\title{
Outer rotor eddy current heater for wind turbines
}

\author{
Tiberiu Tudorache ${ }^{*}$ and Leonard Melcescu \\ University Politehnica of Bucharest, Department of Electrical Machines, Materials and Drives, 313 Splaiul Independentei, \\ 060042 Bucharest, Romania
}

\begin{abstract}
This paper proposes a conversion system of wind energy into thermal energy by means of an outer rotor permanent magnet eddy current heater. The main advantages of this device are the following: compactness, easy temperature control by water flow rate regulation, reduced energy cost per kWh, possibility of combination with other heating systems. The analysis presented in the paper is based on the finite element method and its purpose is to optimize the device and to evaluate its performance and operation characteristics such as: analysis of demagnetization level of permanent magnets, output power-rotor speed and electromagnetic torque-rotor speed curves.
\end{abstract}

\section{Introduction}

Wind energy presents a giant potential for conversion into other forms of useful energies. The most common type of wind systems assures the conversion of wind energy into electricity $[1,2]$. This paper proposes a conversion system of wind energy into thermal energy by means of a permanent magnet eddy current heater (PMECH).

Different solutions of PMECHs have been analyzed so far in [3,4] but in all of them the rotor is placed inside the stator (inner rotor solutions). This paper proposes an outer rotor permanent magnet eddy current heater (ORPMECH) for wind turbines. The main features of this device are the following: compactness, easy control of temperature by water flow rate regulation, reduced energy cost per $\mathrm{kWh}$ and possibility of combination with other heating systems (e.g. thermal solar, photovoltaic thermal, heat pumps, etc.).

The main parts of the proposed device are as follows: an outer rotating part called rotor and an inner fixed part called stator, the two parts being separated by an airgap.

The rotor consists of several pairs of permanent magnets (PMs) radially magnetized, mounted on the inner surface of a magnetic core of hollow cylinder shape, Figure 1(a). On the outer surface of the rotor magnetic core the wind turbine hub can be directly mounted, leading thus to important space savings.

The stator is placed inside the rotor and it consists mainly of a tubular serpentine with thick walls made of magnetic steel mounted on the pole of the wind turbine, Figure 1(b) and (c). The serpentine is thermally insulated on the outer surface so as to reduce the thermal losses and to prevent the heat from migrating to the rotor PMs.

Driven by the rotary motion of the wind turbine the PMs mounted on the ORPMECH rotor will generate eddy currents in the stator serpentine walls heating them up by

\footnotetext{
* e-mail: tiberiu.tudorache@upb.ro
}

Joule effect. The generated heat will be then transferred to the fluid thermal agent that flows through the tubular serpentine (the fluid enters the serpentine in cold state and exists in hot state). The temperature of the fluid will increase and the resulting thermal energy will become available for other useful purposes. The fluid temperature must be controlled electronically so as to keep the PMs of the ORPMECH cool enough to prevent irreversible demagnetization.

The main objectives of this paper refer to the analysis of the proposed ORPMECH from electromagnetic point of view to optimize its structure and to evaluate its performance and operation characteristics. The numerical investigations will be carried out using the finite element (FE) method implemented in the Flux professional software package (Flux ${ }^{\circledR}$ by Cedrat).

\section{Numerical model used for the ORPMECH analysis}

Taking into account the geometry and the specific electromagnetic field structure of the ORPMECH, the analysis of the device can be done properly by a $2 \mathrm{D} F E$ numerical approach with parallel symmetry. In this hypothesis, by taking into account the physical symmetries, the $2 \mathrm{D}$ computation domain will be limited to a single magnetic pole of the device, Figure 2.

The main dimensions of the ORPMECH are the following: outer diameter of rotor core $62.3 \mathrm{~mm}$, inner diameter of rotor core $55.3 \mathrm{~mm}$, outer diameter of steel serpentine $50 \mathrm{~mm}$, permanent magnet arc angle $75 \%$ of pole pitch, permanent magnet thickness $5 \mathrm{~mm}$. The rated speed of the device is $200 \mathrm{rpm}$ and the rated power is $1 \mathrm{~kW}$ (thermal power). Considering $5 \%$ thermal losses the rated induced power will be $P_{\text {indn }}=$ $1.053 \mathrm{~kW}$. 


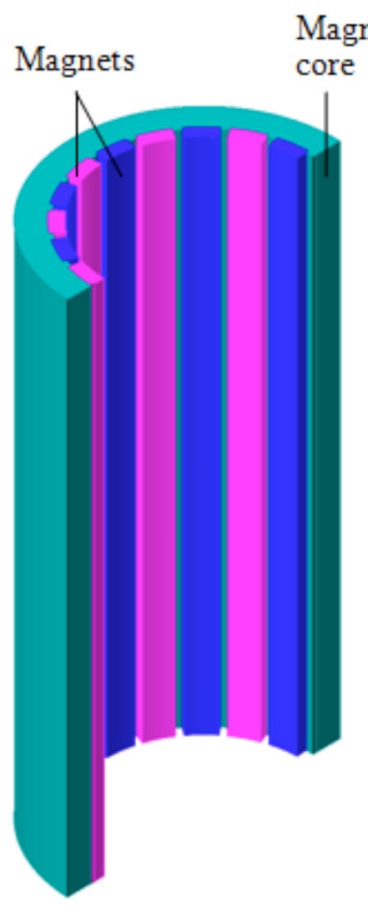

a)

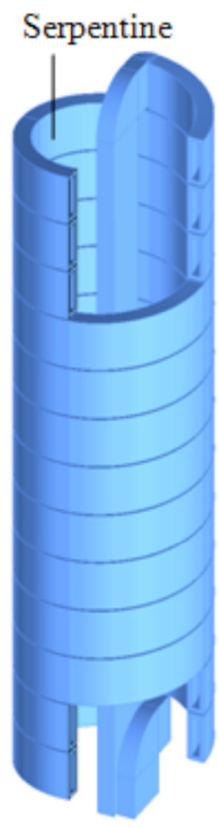

b)

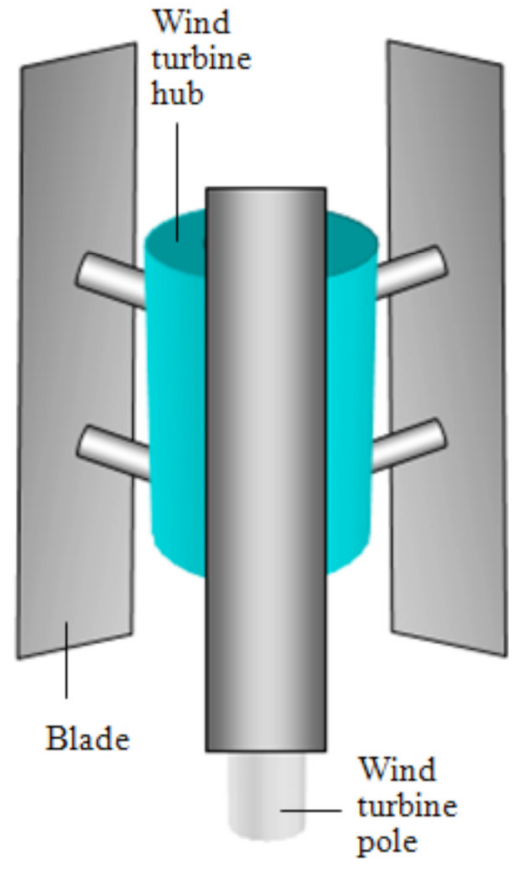

c)

Fig. 1. ORPMECH for wind turbines; (a) outer rotor of the device equipped with PMs; (b) inner stator of the device (steel tube serpentine); (c) vertical axis wind turbine with its hub mounted directly on the outer rotor of the device.
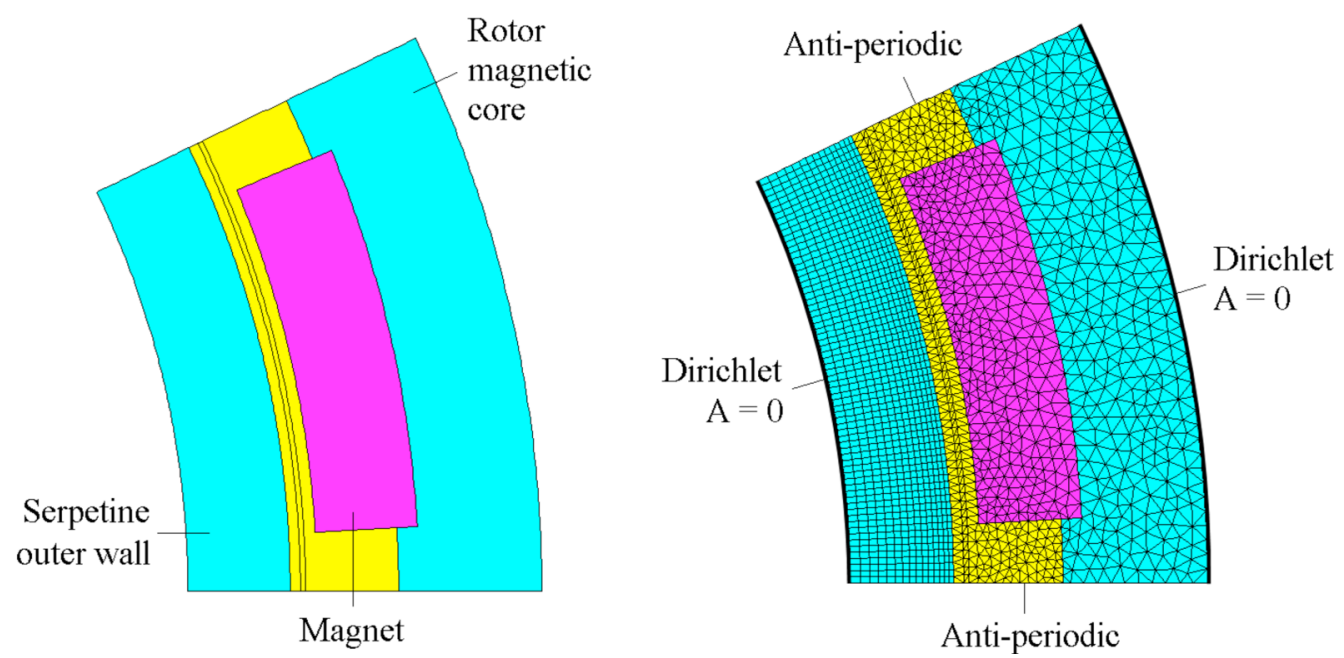

Fig. 2. Computation domain of $2 \mathrm{D}$ FE analysis, mesh and boundary conditions.

The investigation of the ORPMECH from electromagnetic field point of view is done by a $2 \mathrm{D}$ transient magnetic analysis characterized by the following partial differential equation expressed in magnetic vector potential $\boldsymbol{A}[5]$ :

$$
\operatorname{curl}\left[(1 / \mu) \operatorname{curl} \boldsymbol{A}-\boldsymbol{H}_{c}\right]=-\sigma \partial \boldsymbol{A} / \partial t,
$$

where $\mu$ is the magnetic permeability, $\boldsymbol{H}_{c}$ is the coercive field strength of PMs and $\sigma$ is the electrical conductivity of solid conductors.
The boundary conditions imposed on the outer frontier of the rotor and inner frontier of the stator is of Dirichlet type $(\boldsymbol{A}=0)$ and on the radial axes are of anti-periodic type.

The material properties used for the FE simulations are as follows: electrical resistivity of steel $\rho_{\mathrm{s}}=1.6 \times 10^{-7} \Omega \mathrm{m}$, saturation magnetic flux density of steel $B_{\mathrm{s}}=1.9 \mathrm{~T}$, initial relative magnetic permeability of steel $\mu_{\mathrm{r}}=500$, PMs of $\mathrm{NdFeB}$ type with relative magnetic permeability $\mu_{\mathrm{rm}}=$ 1.038 , remnant flux density $B_{\mathrm{r}}=1.28 \mathrm{~T}$ and electrical resistivity $\rho_{\mathrm{m}}=1.6 \times 10^{-6} \Omega \mathrm{m}$. 


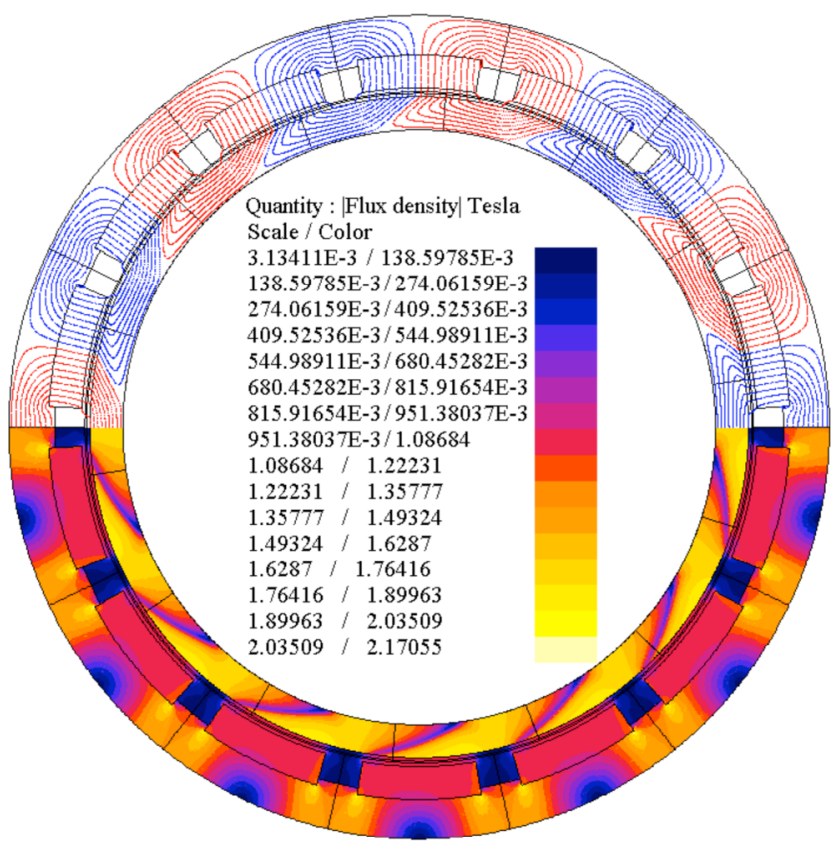

Fig. 3. Equi-flux lines and magnetic flux density distribution on the ORPMECH cross section.

\section{Device optimization and evaluation of operation characteristics}

The main purpose of the transient magnetic analysis is to optimize the device and to evaluate its main operation characteristics represented by the output power-speed and output torque-speed curves. These characteristics are very important for the proper design of the wind turbine used to drive the ORPMECH. If these curves are not taken into account a high risk of blocking the wind turbine at low speeds may arise.
Another important aspect considered during the electromagnetic analysis consists in the evaluation of the magnetization state of the PMs so as to avoid their irreversible demagnetization. The magnetization state of PMs is justified by the fact that the operation of the device is similar to an overloaded PM synchronous generator, which means important demagnetization fields that may affect drastically the operation point of PMs. The heat developed in the device may also contribute to the PMs demagnetization.

After a series of transient magnetic simulations for different operation conditions of ORPMECH we obtained the numerical results shown in Figures 3-6. In Figure 3 are presented the equi-flux lines and the distribution of magnetic flux density on a cross-section through the device. We can notice that the values of the magnetic flux density in the PMs do not descend below critical values (no risk of PMs demagnetization).

The influence of poles number on the induced power is very important for the optimization of the device. According to the results shown in Figure 4, the optimum number of poles corresponding to the largest value of the induced power $\left(P_{\text {ind }} \cong 1.101 \mathrm{~kW}\right)$ is $2 p=14$. These results correspond to an axial length of the device of $400 \mathrm{~mm}$. In order to obtain the rated value of the induced power $\left(P_{\text {ind }}=1.053 \mathrm{~kW}\right)$ the axial length should be cut down to about $383 \mathrm{~mm}$. The numerical results presented in Figures 5 and 6 will correspond to this optimum axial length of $383 \mathrm{~mm}$ and to a number of poles $2 p=14$.

The transient analysis results obtained for different angular speeds of the rotor are shown in Figures 5 and 6 . These results reveal practically a quadratic dependence of the induced power $P_{\text {ind }}[\mathrm{W}]$ on the rotor speed $n[\mathrm{rpm}]$ and a linear dependence of the electromagnetic torque $T_{\mathrm{e}}[\mathrm{Nm}]$ on the rotor speed $n[\mathrm{rpm}]$. These two curves are important for the wind turbine design and they can be approximated by

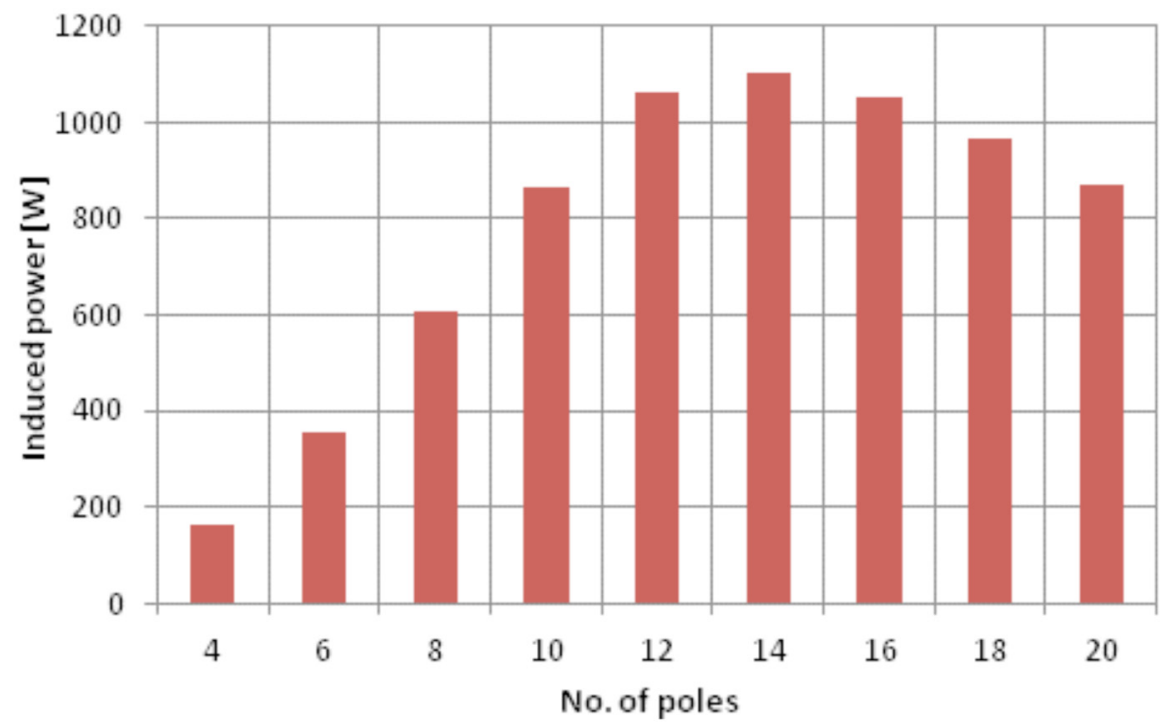

Fig. 4. Influence of number of poles on the induced power of ORPEMCH. 


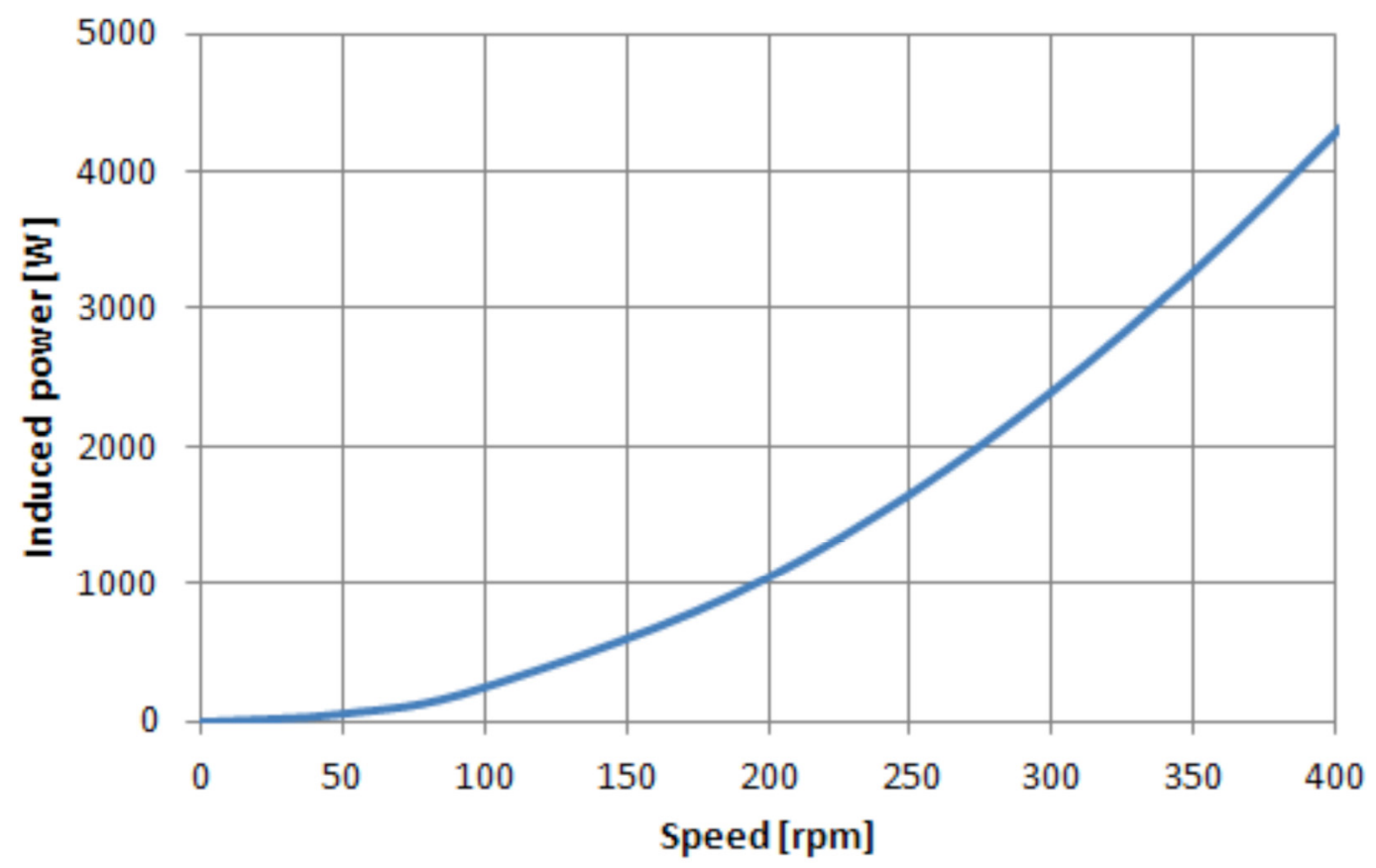

Fig. 5. Power-speed curve of ORPEMCH.

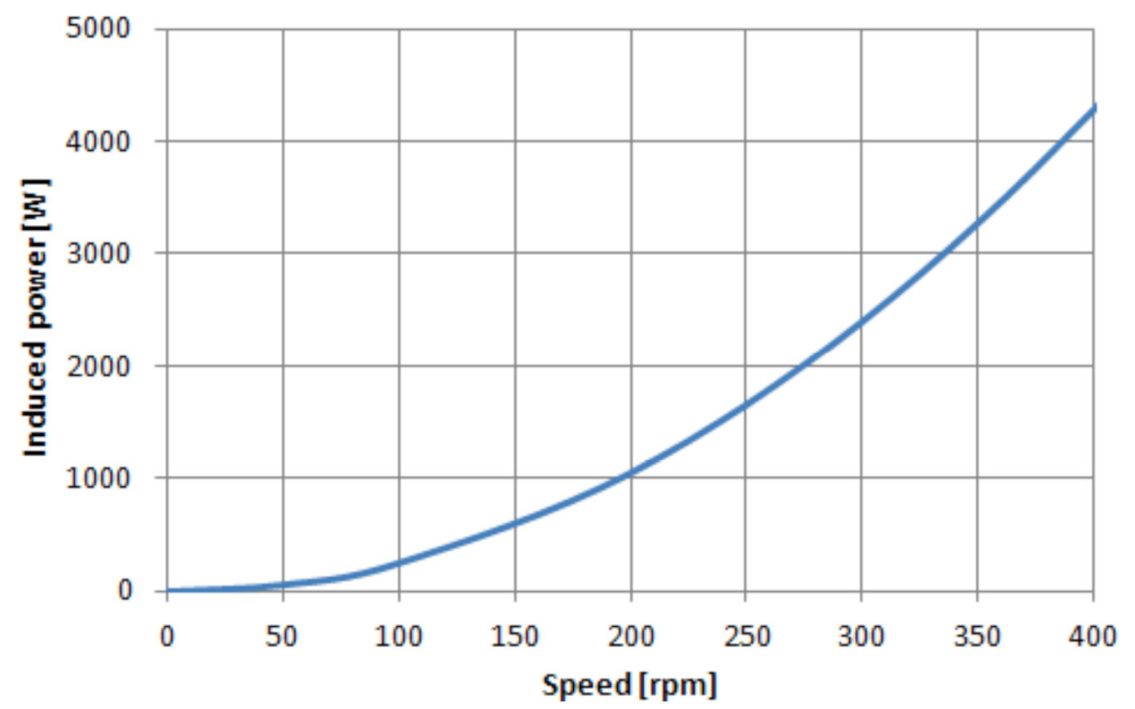

Fig. 6. Electromagnetic torque-speed curve of ORPEMCH.

the following analytical functions:

$$
\begin{gathered}
P_{\text {ind }}=0.0264 n^{2}+0.1195 n, \\
T_{e}=0.254 n .
\end{gathered}
$$

\section{Conclusions}

This paper presents the FE analysis of an ORPMECH from electromagnetic point of view with the aim of optimizing its structure and evaluating its performance and operation characteristics.
The numerical investigations allowed us to identify the optimum number of poles $(2 p=14)$ leading to the maximum induced power in the stator serpentine walls, to evaluate the magnetization state of the PMs and to determine the output power-speed and electromagnetic torque-speed curves of the device. These curves are very important for a proper design of the wind turbine used to drive the ORPMECH.

This paper was elaborated in the framework of the Programme Partnership in prioritary domains - PNII, financed by MEN UEFISCDI, project no. $41 / 2014$. 


\section{References}

1. N. Goudarzi, W.D. Zhu, A review on the development of wind turbine generators across the world, Int. J. Dynam. Control 1, $192(2013)$

2. L. Barote, C. Marinescu, Modeling and operational testing of an isolated variable speed PMSG wind turbine with battery energy storage, Adv. Electr. Comput. Eng. 12, 81 (2012)
3. O. Nebi, V. Fireteanu, Finite element analysis of an eddy current heater for wind or water kinetic energy conversion into heat, in Proceedings of ICREPQ 2010 (2009), p. 506

4. T. Tudorache, M. Popescu, FEM optimal design of wind energy-based heater, Acta Polytech. Hung. 6, 55 (2009)

5. T. Tudorache, L. Melcescu, PMSGs solutions for gearless wind conversion systems with battery storage, in Proceedings of ICREPQ 2013 (2013), p. 479

Cite this article as: Tiberiu Tudorache, Leonard Melcescu, Outer rotor eddy current heater for wind turbines, Renew. Energy Environ. Sustain. 1, 25 (2016) 